\title{
Lattice dynamics in spin-crossover nanoparticles through nuclear inelastic scattering
}

\author{
Gautier Félix, ${ }^{1,2}$ Mirko Mikolasek, ${ }^{1,2}$ Haonan Peng, ${ }^{1}$ William Nicolazzi, ${ }^{1}$ Gábor Molnár, ${ }^{1}$ Aleksandr I. Chumakov, ${ }^{3}$ \\ Lionel Salmon, ${ }^{1}$ and Azzedine Bousseksou ${ }^{1, *}$ \\ ${ }^{1}$ Laboratoire de Chimie de Coordination, CNRS UPR-8241 and Université de Toulouse, UPS, INP, Toulouse, France \\ ${ }^{2}$ Institut Charles Gerhardt, Université Montpellier 2, place Eugène Bataillon, Montpellier, France \\ ${ }^{3}$ ESRF-The European Synchrotron, CS40220, 38043 Grenoble Cedex 9, France \\ (Received 7 November 2014; revised manuscript received 8 January 2015; published 22 January 2015)
}

\begin{abstract}
We used nuclear inelastic scattering (NIS) to investigate the lattice dynamics in [Fe(pyrazine)(Ni(CN) $\left.\left.)_{4}\right)\right]$ spin crossover nanoparticles. The vibrational density of states of iron was extracted from the NIS data, which allowed to determine characteristic thermodynamical and lattice dynamical parameters as well as their spin-state dependence. The optical part of the NIS spectra compares well with the Raman scattering data reflecting the expansion/contraction of the coordination octahedron during the spin transition. From the acoustic part, we extracted the sound velocity in the low-spin $\left(v_{\mathrm{LS}}=2073 \pm 31 \mathrm{~m} \mathrm{~s}^{-1}\right)$ and high-spin $\left(v_{\mathrm{HS}}=1942 \pm 23 \mathrm{~m} \mathrm{~s}^{-1}\right)$ states of the particles. The spin-state dependence of this parameter is of primary interest to rationalize the spin-transition behavior in solids as well as its dynamics and finite size effects.
\end{abstract}

DOI: 10.1103/PhysRevB.91.024422

PACS number(s): 64.70.Nd, 63.22.-m, 75.30.Wx, 76.80.+y

\section{INTRODUCTION}

Molecular spin-crossover (SCO) complexes have received much attention because of their possible applications in memory and display devices and as molecular switches [1]. Indeed, the SCO phenomenon is an exciting example of molecular bistability in which a transition metal ion with a $3 d^{4}-3 d^{7}$ configuration can exhibit a reversible switching between the molecular low-spin (LS) and high-spin (HS) states upon the application of an external stimulus such as temperature, pressure, intense magnetic field, or light irradiation. This phenomenon is accompanied by a spectacular modification of the magnetic, optical, electrical, and mechanical properties of the material. Due to the strong electron-lattice coupling, the molecule occupies a smaller volume in the LS state, which implies a higher density and stiffness when compared to the HS form. In bulk SCO solids, this misfit between the HS and LS molecular volumes leads to strong elastic interactions, which play a major role in the cooperativity of spin transition [2] and its spatiotemporal dynamics [3,4]. In addition, new attractive applications of SCO materials such as microactuators [5,6], magnetostrictive heterostructures [7], or bistable composites [8] are based on the important spontaneous strain accompanying the SCO. Hence the knowledge of the elastic constants of these materials and their spin-state dependence is of significant importance. Unfortunately, the lattice dynamics and, in particular, the acoustic phonon modes of SCO materials remain largely unknown and their elastic constants have been determined only in a few occasions (and usually in only one spin state), using AFM [9], x-ray diffraction [10,11], and Brillouin spectroscopy [12]. In this context, nuclear inelastic scattering (NIS) is a very suitable technique [13], which allows to extract many lattice dynamical parameters and this even in nano-objects, which are of current interest in the SCO field.

The phenomenon underlying this technique is the emission or absorption of a $\gamma$-ray photon, without loss of energy due

\footnotetext{
*azzedine.bousseksou@1cc-toulouse.fr
}

to recoil of the nucleus and without thermal broadening, well-known as the Mössbauer effect [14]. In conventional Mössbauer spectroscopy, the information about the mechanical properties is summarized in the Lamb-Mössbauer factor $f_{\mathrm{LM}}$, associated to the fraction of nuclear resonant absorption. $f_{\mathrm{LM}}$ is a lattice dynamical parameter, which can be related to the lattice stiffness of the material. Conventional Mössbauer spectroscopy is an important technique to characterize $\mathrm{Fe}$ based SCO compounds [15,16], and it was also used at a few occasions to investigate their lattice dynamics through the measurement of $f_{\mathrm{LM}}[17,18]$. On the other hand, the NIS method can provide the complete $57-\mathrm{Fe}$ absorption spectrum in solids, liquids, or gases. However, as in Raman spectroscopy, the Stokes and anti-Stokes peaks are very weak compared to the Rayleigh peak and the intensity of conventional $\gamma$-ray sources is not strong enough to observe them. Currently, the only source that enables to overcome this problem is the synchrotron radiation. In the SCO field, NIS has already been used to characterize the optical modes [19-22], but this technique has not yet been used to study the acoustic modes, which can give access to the vibrational density of states (DOS) at low energies [13] and thereby, provide some important lattice dynamical parameters and their spin-state dependence.

In this paper, we use the NIS technique to investigate the lattice dynamics in SCO nanoparticles and especially the acoustic phonon modes. The sound velocity in the two spin states is extracted from the low-energy part of the DOS and we deduce also the associated Young's and bulk modulus changes. The experiment is performed at the Nuclear Resonance beamline ID18 in $7 / 8+1$ bunch mode at the European Synchrotron Radiation Facility (ESRF) using 50-nm [Fe(pyrazine $\left.)\left(\mathrm{Ni}(\mathrm{CN})_{4}\right)\right]$ nanoparticle sample [23] fully enriched with $57-\mathrm{Fe}$, which presents an abrupt spin transition around $284 \mathrm{~K}$ with an hysteresis loop of $4 \mathrm{~K}$ (see Fig. 1).

\section{RESULTS AND DISCUSSION}

Variable temperature NIS spectra were collected and processed as described in Ref. [13] to obtain the iron DOS of $\left[\mathrm{Fe}(\right.$ pyrazine $\left.)\left(\mathrm{Ni}(\mathrm{CN})_{4}\right)\right]$. In the general case, it is the 
(a)
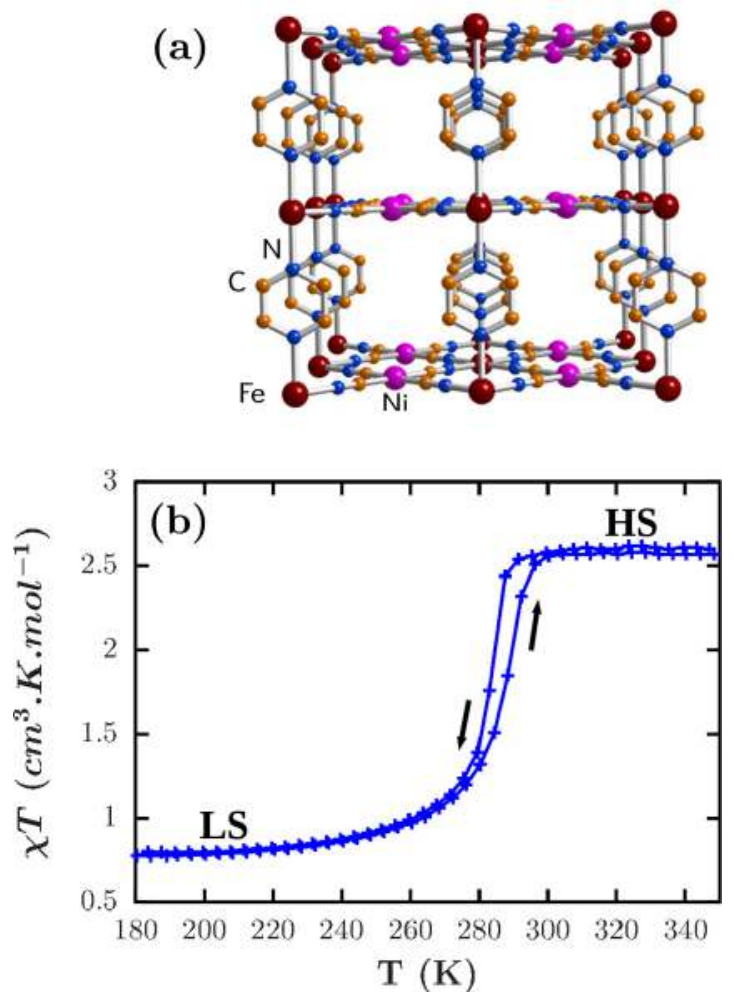

FIG. 1. (Color online) (a) Perspective view of the structure of the $\left[\mathrm{Fe}(\right.$ pyrazine $\left.)\left(\mathrm{Ni}(\mathrm{CN})_{4}\right)\right]$ complex and $(\mathrm{b})$ the product of the magnetic susceptibility and the temperature as a function of the temperature in the heating and cooling modes for $50 \mathrm{~nm}\left[\mathrm{Fe}(\right.$ pyrazine $\left.)\left(\mathrm{Ni}(\mathrm{CN})_{4}\right)\right]$ particles.

projected iron DOS that is obtained. Indeed, the vibrational polarizations are projected onto the incident photon direction, and in particular, the vibrational modes with polarization perpendicular to the direction of the incident photon do not contribute. In our case, the measured vibrational properties are an average over all directions because the sample is a powder. Then, the obtained spectrum is independent on the incident photon direction and the projected DOS and the real DOS are the same. Figures 2(a) and 2(b) show, respectively, the NIS spectra and the vibrational DOS of 50-nm $\left[\mathrm{Fe}(\right.$ pyrazine $\left.)\left(\mathrm{Ni}(\mathrm{CN})_{4}\right)\right]$ nanoparticles in the two spin states (50 K: LS, $310 \mathrm{~K}$ : HS). In the LS state, several Fe-ligand vibrational modes are observed between 20 and $70 \mathrm{meV}$. In the HS state, one large peak is observed around $30 \mathrm{meV}$. This latter is clearly composed of several modes, which could not be resolved. As expected, the vibrational frequencies in the LS state are higher than in the HS state. This is linked to the volume expansion (approximately 25\%) of the coordination octahedron when going from the LS to the HS state. The Raman spectra (633-nm excitation) of the sample were also collected in the two spin states [Fig. 2(c)]. In the HS (LS) state, Raman modes are observed at 165 (306) and $218(381) \mathrm{cm}^{-1}$, which can be assigned [24] to the $v\left(\mathrm{FeN}_{\text {cycle }}\right)$ and $v\left(\mathrm{FeN}_{\mathrm{NC}}\right)$ metal-ligand stretchings, respectively. There is a reasonably good concordance between the peak positions of the NIS and the Raman spectra. In particular, the NIS data, which imply only the vibrational modes for which the mean-squared displacement of iron ions is significant, provide a direct proof
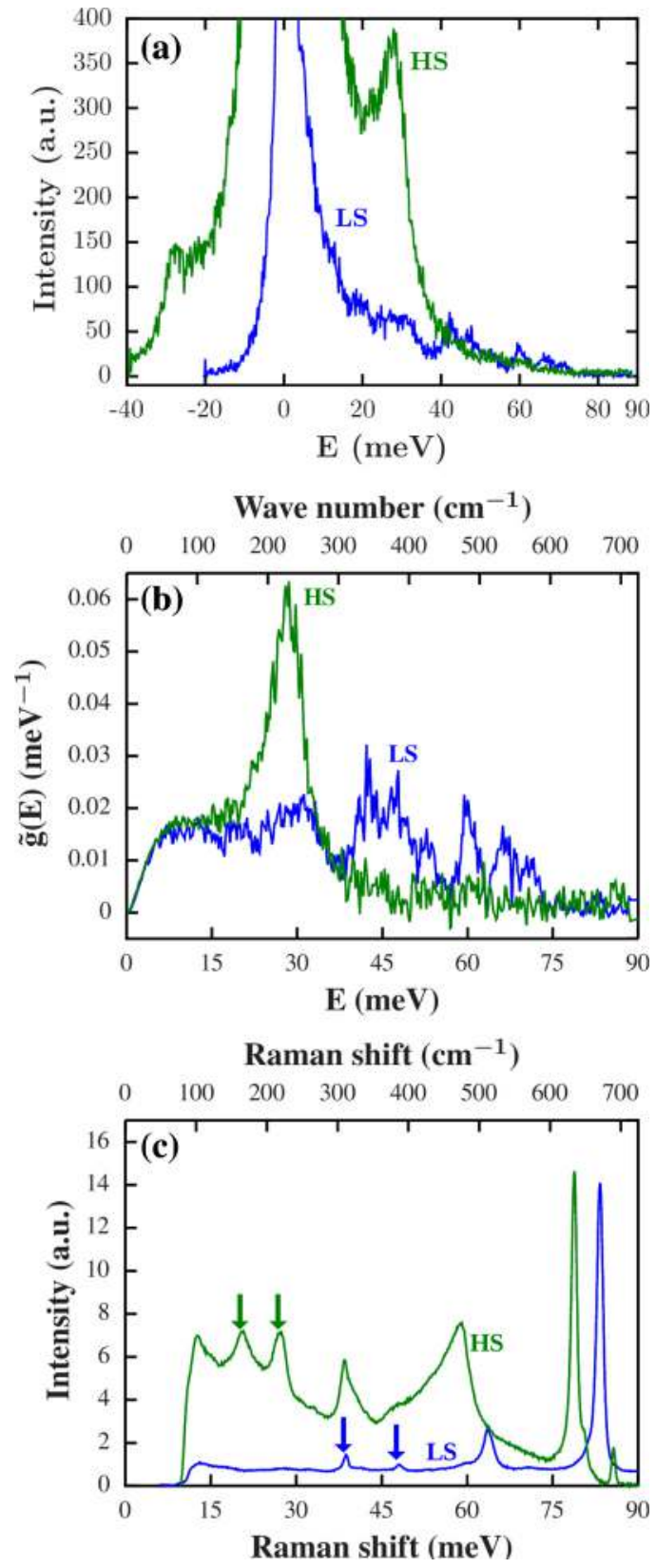

FIG. 2. (Color online) NIS spectra (a), iron-DOS (b), and Raman spectra (c) of 50-nm $\left[\mathrm{Fe}\right.$ (pyrazine) $\left.\left(\mathrm{Ni}(\mathrm{CN})_{4}\right)\right]$ nanoparticles in the two spin states. The central peak of (a) is the elastic absorption, while the peaks on the right and on the left are the Stokes (phonon creation) and the anti-Stokes (phonon annihilation) peaks respectively. Their ratio depends on the temperature. Thereby, in the LS state, the anti-Stokes peaks have a very low intensity when compared to the Stokes peak. In (c), the arrows show, from the left to the right, the $v\left(\mathrm{FeN}_{\text {cycle }}\right)$ and the $v\left(\mathrm{FeN}_{\mathrm{NC}}\right)$ modes in the two spin states.

for the very important increase of the metal-ligand frequencies $\left(v_{\mathrm{LS}} / \nu_{\mathrm{HS}}=1.33\right)$ and the associated mean force constants $\left(C_{\mathrm{LS}} / C_{\mathrm{HS}}=1.7\right)$ when going to the $\mathrm{LS}$ state. These values are consistent with DFT calculations performed on different SCO compounds [19].

Once the phonon DOS is known, various thermodynamical and lattice dynamical parameters can be deduced. Although these parameters may depend on the temperature, we suppose 
TABLE I. Main lattice dynamical parameters extracted from the NIS spectra.

\begin{tabular}{lcc}
\hline \hline & LS $(50 \mathrm{~K})$ & HS $(310 \mathrm{~K})$ \\
\hline Norm. mean force constant $(N / m)$ & 367 & 218 \\
Specific heat & $0.51 k_{B}$ & $2.70 k_{B}$ \\
Entropy & $0.34 k_{B}$ & $3.62 k_{B}$ \\
$f_{\mathrm{LM}}$ & 0.838 & 0.377 \\
$\theta_{D}(\mathrm{~K})$ & 245 & 209 \\
$\sqrt{\left\langle u_{\mathrm{Fe}}^{2}\right\rangle}(\AA)$ & 0.1 & 0.23 \\
\hline \hline
\end{tabular}

the main contribution is due to the spin transition, especially for the $\left[\mathrm{Fe}(\right.$ pyrazine $\left.)\left(\mathrm{Ni}(\mathrm{CN})_{4}\right)\right]$ compound, which does not exhibit significant thermal expansion. Table I summarizes the extracted values. Of particular interest in the SCO field are the specific heat and the entropy. Indeed, the specific heat properties of spin-crossover systems are tightly related to their vibrational properties and the vibrational entropy change is the main driving force of the thermal spin transition. The specific heat, latent heat, and entropy change associated with the SCO is usually determined by calorimetric measurements and the vibrational contribution to the entropy change is calculated as discussed by Ref. [25]. In the compound $\left[\mathrm{Fe}(\right.$ pyrazine $\left.)\left(\mathrm{Ni}(\mathrm{CN})_{4}\right)\right]$, the vibrational entropy change extracted from calorimetry measurements is $55 \mathrm{~J} \mathrm{~K}^{-1} \mathrm{~mol}^{-1}$, but this value was probably underestimated. From Raman spectroscopy, the contribution of the octahedral modes to this entropy change was estimated of about $47 \pm 15 \mathrm{~J} \mathrm{~K}^{-1} \mathrm{~mol}^{-1}$ [24], while from our NIS measurements, we obtain 27 $\mathrm{J} \mathrm{K}^{-1} \mathrm{~mol}^{-1}$ (neglecting the temperature dependence of the entropy). This lower value has two reasons. First, the technique probes only the iron vibrational modes. Nevertheless, because the spin transition occurs in the iron, the most important part of the vibrational entropy change is provided by the iron modes [24]. On the other hand, some modes do not appear due to the fact that NIS technique only probes modes for which $\left\langle u_{\mathrm{Fe}}^{2}\right\rangle \neq 0$. Thereby, the deduced entropy change takes into account only a part of the $\mathrm{FeN}_{6}$ octahedron modes.

From the NIS spectra, the resonant fraction $f_{\mathrm{LM}}$ can be extracted [13]. In general, this procedure does not give the exact value of the Lamb-Mössbauer factor but its upper bound, because the subtracted central elastic peak may hide some unresolved quasielastic contribution to the scattering. The resonant fraction $f_{\mathrm{LM}}$ can be extracted also from the conventional Mössbauer spectra $[14,26]$ or from theoretical simulations $[27,28]$ and is written as $f_{\mathrm{LM}}=\exp \left(-k^{2}\left\langle x^{2}\right\rangle\right)$, where $k$ is the wave vector of the radiation and $\sqrt{\left\langle u^{2}\right\rangle}=$ $\sqrt{3\left\langle x^{2}\right\rangle}$ is the total vibrational amplitude. This latter can be related to the Debye temperature.

From the NIS, the extracted Debye temperatures $\theta_{D}\left(f_{\mathrm{LM}}\right)$ are 245 and $209 \mathrm{~K}$ for the LS and HS states, respectively. On the other hand, for 70-nm nonenriched particles, the Debye temperature has been extracted $\theta_{D}^{\mathrm{LS}}=170 \pm 4 \mathrm{~K}$ from the conventional Mössbauer spectra [23] (in this case, $\theta_{D}$ can be deduced only in the LS state due to the experimental constraints). This difference is not surprising because NIS overestimates $\theta_{D}$ for the same reasons as discussed above for $f_{\mathrm{LM}}$. It is worth to note that the Debye model is

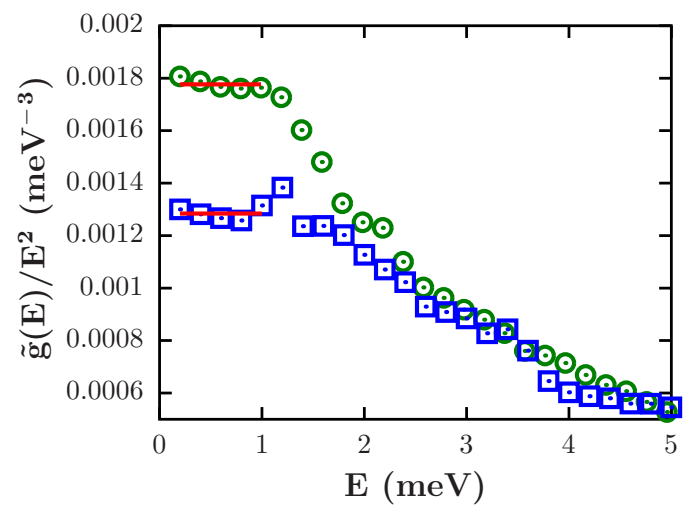

FIG. 3. (Color online) Linear fit (solid line) of $f(E)=\tilde{g}(E) / E^{2}$ for the HS (circle) and the LS (square) states.

intended to describe acoustical modes. Therefore, using the Debye model to characterize the complete phonon spectrum (acoustic and optical modes) of a specific compound leads to the determination of the "effective Debye temperature". In any case, our main interest is the variation of the Debye temperature with the spin state of the system. From the NIS data, we obtain $\theta_{D}^{\mathrm{LS}} / \theta_{D}^{\mathrm{HS}}=1.17$, which indicates a variation of the bulk modulus $B_{\mathrm{LS}} / B_{\mathrm{HS}}=1.37$. An important part of the phonon DOS concerns the acoustic modes, from which the sound velocity can be also extracted. This latter parameter is directly related to the lattice stiffness. In the Debye model and because our sample is a powder, the DOS can be related to the sound velocity as [29]

$$
\frac{\tilde{g}(E)}{E^{2}}=\frac{\tilde{m}}{2 \pi^{2} \rho \hbar^{3} v_{s}^{3}},
$$

where $\hbar$ is the reduced Planck constant, $\tilde{m}$ is the atomic mass of $57-\mathrm{Fe}, \rho$ is the volumetric mass density of $\left[\mathrm{Fe}\right.$ (pyrazine) $\left.\left(\mathrm{Ni}(\mathrm{CN})_{4}\right)\right][30]$ and $v_{s}$ is the Debye sound velocity.

Figure 3 plots $\tilde{g}(E) / E^{2}$ as a function of the energy $E$. The low-energy part of the curves is linear, which corresponds to the Debye model prediction and is therefore proportional to $1 / v_{s}^{3}$. The sound velocity values extracted from the experimental data are $\left(v_{\mathrm{LS}}=2073 \pm 31 \mathrm{~m} \mathrm{~s}^{-1}\right)$ and $\left(v_{\mathrm{HS}}=1942 \pm 23\right.$ $\mathrm{m} \mathrm{s}^{-1}$ ) for the LS and HS states, respectively. Then, the Young's modulus can be deduced from the sound velocity and the Navier's equations as follows (see Appendix):

$$
Y\left(v, v_{s}, \rho\right) \propto v_{s}^{2} \rho .
$$

Although, the Poisson's ratio $v$ of the sample is not known exactly, the Young's modulus weakly depends on it. The Young's modulus was estimated by taking its averaged value for Poisson's ratios between 0.25 and 0.45 . We obtain $\left(Y_{\mathrm{HS}}=\right.$ $10.4 \pm 1.1 \mathrm{GPa})$ and $\left(Y_{\mathrm{LS}}=13.5 \pm 1.3 \mathrm{GPa}\right)$ in the HS and LS states, respectively. The relative change from the HS to the LS state is $Y_{\mathrm{LS}} / Y_{\mathrm{HS}}=1.30 \pm 0.12$. This variation is independent of the actual value of the Poisson's ratio and is in good agreement with the AFM data $\left(Y_{\mathrm{LS}}^{\mathrm{AFM}} / Y_{\mathrm{HS}}^{\mathrm{AFM}}=1.33 \pm 0.04\right)$ obtained for the $\mathrm{SCO}$ complex [ $\left.\mathrm{Fe}^{\mathrm{II}}(\mathrm{hptrz})_{3}\right](\mathrm{OTs})_{2}$ [9]. It may be worth to note that $\frac{B_{\mathrm{HS}} Y_{\mathrm{LS}}}{B_{\mathrm{LS}} Y_{\mathrm{HS}}} \approx 1$. This last result implies the Poisson's ratio is very close in the two spin states $\left(v_{\mathrm{LS}} \approx v_{\mathrm{HS}}\right)$ in this sample. 


\section{CONCLUSION}

In summary, the vibrational DOS and its spin-state dependence have been probed by means of NIS spectroscopy. These spectra revealed the well-known high-frequency shift of the DOS when going from the HS to the LS state. The analysis of the vibrational entropy change accompanying the spin transition has put in evidence the main contribution coming from the metal-ligand vibrational modes. The stiffness change of the material with the spin state change has been analysed through the Lamb-Mössbauer factor and the Debye sound velocity has been also calculated from the acoustic part of the DOS. Using these parameters, the variations of the Young's and the bulk moduli have been estimated in the two spin states. These elastic constants are largely ignored in the spin crossover field and their knowledge is of outmost importance in order to rationalize the origin of the first-order phase transition (cooperativity and associated memory effect) [2] and the ultrafast dynamics [31] in these compounds as well as to develop applications [5-8] based on the spontaneous strain accompanying this transition. A particular interest of the NIS technique is that it can be used to probe the DOS (and thus the elastic constants) of spin crossover nanoparticles and other nano-objects as well. The cooperativity [32] and the applications of these bistable nano-objects are currently in the focus of the spin crossover community and the analysis of the size-dependence of the vibrational DOS and associated material properties (entropy, elasticity, etc.) is obviously a key for the understanding of the spin transition in these nanomaterials and by extension, the understanding of the first-order phase transition with electron-lattice coupling.

\section{ACKNOWLEDGMENTS}

The authors are grateful for the financial support from ANR Nanohybrid (Project No. ANR-13-BS07-0020-01) and for the kind help of José Antonio Real, Leonid Dubrovinsky, and Rudolf Rüffer. H. Peng thanks the China Scholarship Council for a Ph.D. grant.

\section{APPENDIX: YOUNG'S MODULUS}

For an infinite medium, the longitudinal $c_{l}$ and transverse $c_{t}$ sound velocity can be written as

$$
\begin{gathered}
c_{l}=\sqrt{\frac{Y(1-v)}{(1+v)(1-2 v) \rho},} \\
c_{t}=\sqrt{\frac{Y}{2(1+v) \rho}},
\end{gathered}
$$

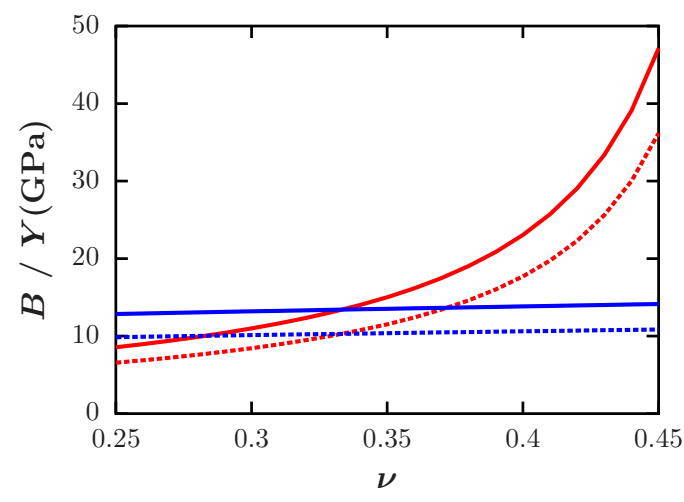

FIG. 4. (Color online) Dependence of the Young's (blue) and bulk (red) modulus with the Poisson's ratio in the HS (dotted line) and LS (straight line) states.

where $Y$ is the Young's modulus, $v$ is the Poisson's ratio, and $\rho$ is the volumetric mass density. The Debye sound velocity is written as

$$
v_{s}=\left(\frac{3 c_{t}^{3} c_{l}^{3}}{2 c_{l}^{3}+c_{t}^{3}}\right)^{\frac{1}{3}}
$$

We can deduce the Young's modulus:

$$
Y=\left(\frac{2 \alpha+\beta}{3}\right)^{\frac{2}{3}} \rho v_{s}^{2},
$$

where,

$$
\alpha=[2(1+v)]^{\frac{3}{2}}, \quad \beta=\left[\frac{(1-2 v)(1+v)}{1-v}\right]^{\frac{3}{2}} .
$$

Then, the bulk modulus can be calculated from

$$
B=\frac{Y}{3(1-2 v)}
$$

Figure 4 shows the Poisson's ratio dependency of the Young's and bulk moduli in the two spin states. While the Young's modulus can be estimated because of its weak dependence on the Poisson's ratio, the change of the bulk modulus is too important to estimate its value.
[1] Spin Crossover in Transition Metal Compounds I, II, III, edited by P. Gütlich and H. A. Goodwin, Topics in Current Chemistry, 233, 234, 235 (Springer, Berlin, Heidelberg, New York, 2004).

[2] H. Spiering, K. Boukheddaden, J. Linares, and F. Varret, Phys. Rev. B 70, 184106 (2004).

[3] W. Nicolazzi and S. Pillet, Phys. Rev. B 85, 094101 (2012).

[4] M. Paez-Espejo, M. Sy, F. Varret, and K. Boukheddaden, Phys. Rev. B 89, 024306 (2014).
[5] H. J. Shepherd, I. A. Gural'skiy, C. M. Quintero, S. Tricard, L. Salmon, G. Molnár, and A. Bousseksou, Nat. Commun. 4, 2607 (2013).

[6] I. A. Gural'skiy, C. Quintero, J. Sanchez Costa, P. Demont, G. Molnár, L. Salmon, H. J. Shepherd, and A. Bousseksou, J. Mater. Chem. C 2, 2949 (2014).

[7] C. R. Gros, M. K. Peprah, B. D. Hosterman, T. V. Brinzari, P. A. Quintero, M. Sendova, M. W. Meisel, and D. R. Talham, J. Am. Chem. Soc. 136, 9846 (2014). 
[8] Y.-S. Koo and J. R. Galán-Mascarós, Adv. Mater. 26, 6785 (2014).

[9] E. M. Hernández, C. M. Quintero, O. Kraieva, C. Thibault, C. Bergaud, L. Salmon, G. Molnár, and A. Bousseksou, Adv. Mater. 26, 2889 (2014).

[10] H. J. Shepherd, P. Rosa, L. Vendier, N. Casati, J.-F. Létard, A. Bousseksou, P. Guionneau, and G. Molnár, Phys. Chem. Chem. Phys. 14, 5265 (2012).

[11] T. Granier, B. Gallois, J. Gaultier, J. A. Real, and J. Zarembowitch, Inorg. Chem. 32, 5305 (1993).

[12] J. Jung, F. Bruchhäuser, R. Feile, H. Spiering, and P. Gütlich, Z. Phys. B 100, 517 (1996).

[13] A. I. Chumakov and W. Sturhahn, Hyperfine Interact. 123-124, 781 (1999).

[14] N. N. Greenwood and T. C. Gibb, Mössbauer Spectroscopy (Chapman and Hall Ltd, London, 1971).

[15] P. Gütlich, H. Köppen, and H. G. Steinhäuser, Chem. Phys. Lett. 74, 475 (1980).

[16] E. König, G. Ritter, and S. K. Kulshreshtha, Chem. Rev. 85, 219 (1985).

[17] A. A. Yousif, H. Winkler, H. Toftlund, A. X. Trautwein, and R. H. Herber, J. Phys.: Condens. Matter 1, 7103 (1989).

[18] Z. Yu, G. Schmitt, S. Hofmann, H. Spiering, Y. F. Hsia, and P. Gütlich, Hyperfine Interact. 93, 1459 (1994).

[19] H. Paulsen, H. Winkler, A. X. Trautwein, H. Grünsteudel, V. Rusanov, and H. Toftlund, Phys. Rev. B 59, 975 (1999).

[20] K. L. Ronayne, H. Paulsen, A. Höfer, A. C. Dennis, J. A. Wolny, A. I. Chumakov, V. Schünemann, H. Winkler, H. Spiering, A. Bousseksou, P. Gütlich, A. X. Trautwein, and J. J. McGarvey, Phys. Chem. Chem. Phys. 8, 4685 (2006).
[21] J. A. Wolny, S. Rackwitz, K. Achterhold, K. Muffler, and V. Schünemann, Hyperfine Interact. 204, 129 (2012).

[22] J. A. Wolny, R. Diller, and V. Schünemann, Eur. J. Inorg. Chem. 2012, 2635 (2012).

[23] H. Peng, S. Tricard, G. Felix, G. Molnar, W. Nicolazzi, L. Salmon, and A. Bousseksou, Angew. Chem. Int. Ed. 53, 10894 (2014).

[24] G. Molnár, V. Niel, A. B. Gaspar, J. A. Real, A. Zwick, A. Bousseksou, and J. J. McGarvey, J. Phys. Chem. B 106, 9701 (2002).

[25] M. Sorai and S. Seki, J. Phys. Chem. Solids 35, 555 (1974).

[26] K. Boukheddaden and F. Varret, Hyperfine Interact. 72, 349 (1992).

[27] M. Mikolasek, G. Félix, G. Molnár, F. Terki, W. Nicolazzi, and A. Bousseksou, Phys. Rev. B 90, 075402 (2014).

[28] H. Paulsen, H. Grünsteudel, W. Meyer-Klaucke, M. Gerdan, H. F. Grünsteudel, A. I. Chumakov, R. Rüffer, H. Winkler, H. Toftlund, and A. X. Trautwein, Eur. Phys. J. B 23, 463 (2001).

[29] M. Y. Hu, W. Sturhahn, T. S. Toellner, P. D. Mannheim, D. E. Brown, J. Zhao, and E. E. Alp, Phys. Rev. B 67, 094304 (2003).

[30] P. D. Southon, L. Liu, E. A. Fellows, D. J. Price, G. J. Halder, K. W. Chapman, B. Moubaraki, K. S. Murray, J.-F. Létard, and C. J. Kepert, J. Am. Chem. Soc. 131, 10998 (2009).

[31] M. Lorenc, J. Hébert, N. Moisan, E. Trzop, M. Servol, M. Buron-Le Cointe, H. Cailleau, M. L. Boillot, E. Pontecorvo, M. Wulff, S. Koshihara, and E. Collet, Phys. Rev. Lett. 103, 028301 (2009).

[32] G. Félix, W. Nicolazzi, L. Salmon, G. Molnár, M. Perrier, G. Maurin, J. Larionova, J. Long, Y. Guari, and A. Bousseksou, Phys. Rev. Lett. 110, 235701 (2013). 